This department welcomes short notes and problems believed to be new. Contributors should include solutions where known, or background material in case the problem is unsolved. Send all communications concerning this department to I. G. Connell, Department of Mathematics, McGill University, Montreal, P.Q.

\title{
GROUPS IN WHICH RAISING TO A POWER IS AN AUTOMORPHISM
}

\section{H. F. Trotter}

For any group $G$ and integer $n$, let $P_{n}: G \rightarrow G$ be the function defined by $P_{n}(g)=g^{n}$ for all $g \in G$. If $G$ is abelian then $P_{n}$ is a homomorphism for all $n$. Conversely, it is well known (and easy to show) that if $P_{2}$ or $P_{-1}$ is a homomorphism then $G$ is abelian. As the groups $G_{n}$ described below show, for every $n$ other than 2 and -1 there exist non-abelian groups for which $P_{n}$ is a homomorphism.

In this note we derive some elementary consequences of the assumption that $P_{n}$ is an automorphism for some particular. value of $n$. One somewhat surprising result is that $P_{3}$ can be an automorphism only if $G$ is abelian.

We begin with some simple lemmas. Let $H(G)$ be the set of integers $n$ such that $P_{n}$ is a homomorphism of $G$, and $A(G)$ the set of integers such that $P_{n}$ is an automorphism of $G$. Since the composition of $P_{n}$ and $P_{m}$ is $P_{m n}$ we have

(1) If $m, n \in H(G)$ then $m n \in H(G)$. 
If $m \notin A(G)$ then the identity $P_{m n}=P_{m} P_{n}$ may be multiplied by $P_{m}^{-1}$ to give $P_{n}=P_{m}^{-1} P_{m n}$. Writing $q$ for $m n$, this gives

(2) If $m \in A(G), q \in H(G)$ and $m$ divides $q$, then $\mathrm{q} / \mathrm{m} \in \mathrm{H}(\mathrm{G})$.

We have $n \in H(G)$ if and only if $h^{n} g^{n}=(h g)^{n}$ for all $h, g \in G$. Setting $h=x^{-1}, g=y^{-1}$, so that $h g=(y x)^{-1}$, converts this identity into $x^{-n} y^{-n}=(y x)^{-n}$. Premultiplication by $x$ and postmultiplication by $y$ gives $x^{1-n} y^{1-n}=(x y)^{1-n}$. Therefore

(3) If $n \in H(G)$ then $1-n \in H(G)$.

Now suppose $n \in A(G)$. By (3), $1-n \in H(G)$, and hence by (1), $(1-n)^{2} \in H(G)$. By (3) again, $1-(1-n)^{2}=2 n-n^{2} \in H(G)$, and by (2), $2-n \in H(G)$. A final application of (3) gives $n-1 \in H(G)$ and we have proved

(4) If $n \in A(G)$ then $n-1 \in H(G)$.

COROLLARY. If $P_{3}$ is an automorphism then $G$ is abelian (since $P_{2}$ is a homomorphism).

LEMMA. If both $n$ and $n+1$ are in $H(G)$, then $k \in H(G)$ implies $k^{\prime} \in H(G)$ for all $k^{\prime} \equiv k(\bmod n)$.

Proof: By as sumption, $g^{\mathrm{n}+1} h^{\mathrm{n}+1}=(\mathrm{gh})^{\mathrm{n}+1}=(\mathrm{gh})^{\mathrm{n}} \mathrm{gh}=$ $\mathrm{g}^{\mathrm{n}} \mathrm{h}^{\mathrm{n}} \mathrm{gh}$ for all $\mathrm{g}, \mathrm{h} \in \mathrm{G}$. Cancelling $\mathrm{g}^{\mathrm{n}}$ on the left and $\mathrm{h}$ on the right gives $\mathrm{gh}^{\mathrm{n}}=\mathrm{h}^{\mathrm{n}} \mathrm{g}$, which shows that all n-th powers are in the centre of $G$. Now suppose $g^{k} h^{k}=(g h)^{k}$ and let $r$ be any integer. We have $g^{k+n r_{h}{ }^{k+n r}}=g^{k} h^{k}\left(g^{n} h^{n}=\right.$ $(g h)^{k}\left((g h)^{n}\right)^{r}=(g h)^{k+n r}$, using the facts that $h^{n}, g^{n}$ are in the centre of $G$ and that $n \in H(G)$. 
THEOREM. If $n+1 \in A(G)$ then $H(G)$ consists of the union of congruence classes modulo $n$, and contains at least all integers congruent to 0 or 1 modulo $n$.

Proof: By (4) (with $\mathrm{n}+1$ in place of $\mathrm{n}$ ) the hypothesis of the Iemma is satisfied. Obviously 0 and 1 are in $H(G)$ for any group $G$.

A sequence of examples $G_{n}$ with $n+1 \in A\left(G_{n}\right)$ which exhibits some non-trivial possibilities for the set $H(G)$ may be defined as follows. The elements of $G_{n}$ are triples $(x, y, z)$ of integers modulo $n$ (so $G_{n}$ has order $n^{3}$ ) and multiplication is defined by $(x, y, z)\left(x^{\prime}, y^{\prime}, z^{\prime}\right)=\left(x+x^{\prime}, y+y^{\prime}, z+z^{\prime}+2 x y^{\prime}\right)$. The group is non-abelian for $n>2$. An easy induction shows that $(x, y, z)^{k}=(k x, k y, k z+k(k-1) x y)$. Thus $P_{n+1}$ is the identity map and $n+1 \in A\left(G_{n}\right)$. Direct calculation shows that $k \in H\left(G_{n}\right)$ if and only if $k(k-1)$ is divisible by $n$, which is consistent with the conclusion of the theorem.

Princeton University 